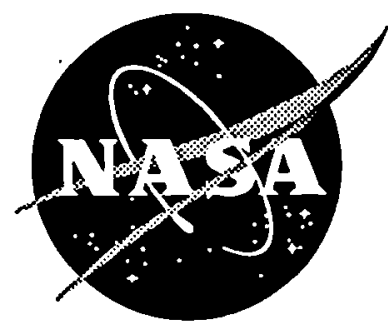

\title{
Separation of Crack Extension Modes in Orthotropic Delamination Models
}

Jack L. Beuth

Carnegie Mellon University, Pittsburgh, Pennsylvania

April 1995

National Aeronautics and Space Administration Langley Research Center

Hampton, Virginia 23681-0001

\section{LIBRARY COPY}

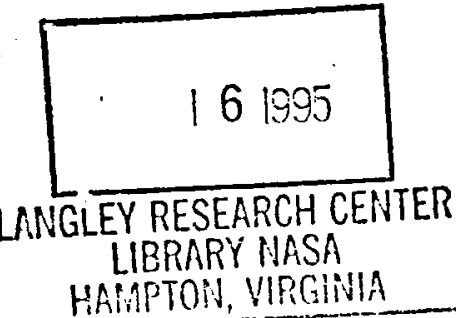




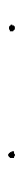

. 


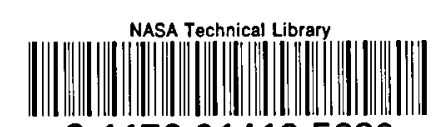

31176014195680

\title{
Separation of Crack Extension Modes \\ in Orthotropic Delamination Models
}

\author{
Jack L. Beuth \\ Assistant Professor \\ Department of Mechanical Engineering \\ Carnegie Mellon University \\ Pittsburgh, PA 15213
}

\begin{abstract}
In the analysis of an interface crack between dissimilar elastic materials, the mode of crack extension is typically not unique, due to oscillatory behavior of near-tip stresses and displacements. This behavior currently limits the applicability of interfacial fracture mechanics as a means to predict delamination in layered materials. The Virtual Crack Closure Technique (VCCT) is a method used to extract mode I and mode II energy release rates from numerical fracture solutions. The mode of crack extension extracted from an oscillatory solution using the VCCT is not unique due to the dependence of mode on the virtual crack extension length, $\Delta$.

In this work, a method is presented for using the VCCT to extract $\Delta$-independent crack extension modes for the case of an interface crack between two in-plane orthotropic materials. The method does not involve altering the analysis to eliminate its oscillatory behavior. Instead, it is argued that physically reasonable, $\Delta$-independent modes of crack extension can be extracted from oscillatory solutions. Knowledge of near-tip fields is used to determine the explicit $\Delta$ dependence of energy release rate parameters. Energy release rates are then defined that are separated from the oscillatory dependence on $\Delta$. A modified VCCT using these energy release rate definitions is applied to results from finite element analyses, showing that $\Delta$-independent modes of crack extension result. The modified technique has potential as a consistent method for extracting crack extension modes from numerical solutions. The $\Delta$-independent modes extracted using this technique can also serve as guides for testing the convergence of finite element models. Direct applications of this work include the analysis of planar composite delamination problems, where plies or debonded laminates are modeled as in-plane orthotropic materials.
\end{abstract}

\section{Introduction}

The problem of delamination in composite materials has been studied extensively in the literature. From this work, a methodology for analyzing delamination problems has emerged, where the problem is treated within the framework of fracture mechanics. In order to predict delamination resistance, the problem of an existing interfacial delamination crack is modeled, and an applied stress intensity factor, $\mathrm{K}$, or energy release rate, $\mathrm{G}$, is calculated. The applied $\mathrm{K}$ or $\mathrm{G}$ is then compared to a mode-dependent critical value of $\mathrm{K}$ or $\mathrm{G}$ from experiment. The work 
of Wang and Crossman (1980), O'Brien (1982) and Wang (1982) was some of the earliest to use this type of approach. One advantage of a fracture-based approach is that finite energy release rates result, even for limiting cases where the length of the delamination crack approaches zero. In contrast, if a fully bonded interface is modeled, singular stresses result at the intersection of the interface and the free edge. This makes prediction of delamination through use of a fully bonded model problematic.

The mode dependence of critical $\mathrm{K}$ or $\mathrm{G}$ values requires that an applied stress intensity factor be compared to a measured interfacial toughness for the same mode of crack extension. Commonly used interfacial toughness tests include the double cantilever beam test, which yields a pure mode I interfacial toughness and the end notched flexure test, which yields a toughness close to that for pure mode II (see Fig. 1). Other tests have been proposed to measure toughesses under various mixed mode conditions e.g. the work of Reeder and Crews (1990). Composite interfacial toughness tests are typically performed on unidirectional $0^{\circ}$ laminates. Because the elastic properties of adjacent plies are the same, the mode of crack extension associated with such tests can be unambiguously determined. In contrast, in most applications, delaminations occur between plies of different orientation. In such cases, modeling the delamination as a crack along a distinct interface between plies typically results in near-tip stresses and displacements that oscillate as the tip is approached. As a result, in the interfacial fracture analysis of most composite applications, the mode of crack extension is a function of distance from the crack tip and is not uniquely defined. Under such conditions, a direct match of mode between the application and coupon tests cannot readily be made. A method is needed for extracting crack extension modes from interfacial fracture analyses that exhibit oscillatory behavior. In this study, a method is proposed for extracting physically relevant crack extension modes from oscillatory analyses where the materials defining the interface are modeled as in-plane orthotropic materials.

\section{Background}

A number of methods have been proposed for extracting fracture modes in interfacial fracture analyses that exhibit oscillatory behavior. Two existing approaches involve altering the analysis to eliminate the oscillatory nature of the solution. Once this is done, a unique mode of crack extension can be extracted. The first of these methods is often termed the resin interlayer method and was originally proposed for analyzing isotropic fracture problems by Atkinson (1977). In its most commonly used form, it involves inserting a thin homogeneous layer (the resin interlayer) between the layers forming the interface and placing the crack within it. Because the crack tip is fully embedded in a homogeneous material, non-oscillatory stresses and displacements result. 
The resin interlayer method has some appeal in modeling delamination in resin matrix composites because they have a resin-rich region between adjacent plies. This suggests that the inclusion of a resin interlayer in the modeling is physically reasonable. The method has disadvantages, however. First, the additional effort required to apply the method acts as a deterrent to the method's widespread use. Significantly more effort is required in finite element analyses to mesh out the interlayer region. Another disadvantage of the resin interlayer method is the addition of the interlayer thickness and properties as variables in the problem. How sensitive the results of the analysis are to relatively small changes in the modeling of the interlayer is not clear. A final disadvantage of this method involves its true relation to the physical delamination problem on an interlayer scale. As Atkinson (1977) himself notes, placing the crack fully within the interlayer ignores the fact that a crack under mixed mode conditions in a homogeneous material will not grow in a self-similar manner. In the general case of mixedmode loading, a crack within an interlayer will not remain there as it grows, but will branch to an interface. On an interlayer scale, modeling a delamination as the extension of a single crack may also be non-physical. The work of Chai (1992) suggests that macroscopically mode II dominated crack extension in brittle interlayers occurs by the growth of pure mode I microcracks ahead of the tip which then link up with the main crack tip.

A second method of approaching the interface delamination problem involves changing "physically insignificant" properties of one or both layers forming the interface to make the oscillatory exponent parameter, $\varepsilon$, equal zero (definitions for $\varepsilon$ are given in the next section). This method, outlined for orthotropic analyses in the work by Davidson (1993), typically involves changing a Poisson's ratio of one of the layers forming the interface. It is based on an analogous method proposed for isotropic bimaterial fracture problems by He and Hutchinson (1989) and Suo and Hutchinson (1989). The key to this method is the identification of "physically insignificant" properties that can be changed. For the isotropic bimaterial case, it is well-established that most interfacial fracture problems are very weakly dependent on $\varepsilon$ or the related Dundurs parameter $\beta$. Thus in most analyses of isotropic bimaterials, it is possible to change properties of one or more of the layers forming the interface to make $\varepsilon=0$ without significantly altering the physics of the analysis. An analogous role for the parameter $\varepsilon$ for orthotropic and anisotropic interface problems has not been established. Although this method has potential, standardized application of it requires formulation of consistent criteria for identifying which properties can be altered to make $\varepsilon=0$ without significantly changing the physical aspects of the model.

A third method for extracting consistent modes of crack extension from oscillatory analyses does not involve altering the interfacial fracture analysis. Instead, it is recognized that severe mode perturbations due to oscillatory effects are confined to a region very close to the 
crack tip. Outside of this region, the mode of crack extenision is only weakly dependent on distance from the crack tip. An argument is made that physically reasonable modes of crack extension can be extracted from an unaltered interfacial fracture analysis at a prescribed distance from the crack tip that is outside of the zone dominated by $\varepsilon$ effects. The concepts associated with this method were originally detailed by Rice (1988) for the case of an interface crack between isotropic layers. This method has been applied by numerous researchers to composite delamination problems using the Virtual Crack Closure Technique (VCCT), where a virtual crack extension length, $\Delta$, is used that is large compared to the zone dominated by $\varepsilon$ effects (see O'Brien (1982), for example). This method is appealing because it does not require that the analysis itself be altered. It results in reasonable and consistent modes of crack extension if a consistent value of $\Delta$ is used from problem to problem. The principal drawbacks of this method are related to its application. The oscillatory nature of the interfacial fracture solution places a limit on how close to the crack tip the VCCT can be applied. However, as with other "local fitting" methods, it is important to apply the VCCT in a region close to the crack tip where the near-tip (singular) fields dominate. Use of numerical results to identify a value of $\Delta$ satisfying both of these requirements can be difficult, particularly if it is coupled with the task of obtaining a finite element solution that is sufficiently refined with respect to extracting crack extension mode.

Any of the three methods outlined thus far can, if carefully applied, provide useful mode values for interfacial fracture problems. However, each method currently has drawbacks related to the ease and/or consistency with which it can be applied by practitioners. In the current study, it is argued that practitioners will not want to alter interfacial fracture analyses (e.g. by changing properties or by inserting a resin layer). Altering analyses requires increased effort and standard methods for altering analyses have not been defined. As in the third method outlined above, attention is focused on extracting a physically reasonable mode of crack extension from the existing oscillatory analysis. This is done by using asymptotic near-tip stress and displacement relations to define a mode that is separated from oscillatory effects. This definition is proposed as a consistent measure of crack extension mode for orthotropic interfacial fracture problems.

\section{Theory}

\section{Isotropic interfacial fracture}

Before the problem of an interfacial crack between orthotropic layers is addressed, the problem of an interfacial crack between isotropic layers will be briefly outlined. Although the equations associated with the orthotropic problem are more complicated, the conceptual problems associated with the isotropic and orthotropic problems are the same. Expressions given 
for stress intensity factors and near-tip stress fields follow those used by (among others) Rice (1988) and Suo and Hutchinson (1990).

For a crack along the interface between two isotropic layers (see Fig. 2), the singular stresses just ahead of the interfacial crack tip (along the positive $\mathrm{x}$ axis) take the following form:

$$
\sigma_{\mathrm{yy}}+\mathrm{i} \sigma_{\mathrm{xy}}=\frac{\mathrm{K}}{\sqrt{2 \pi \mathrm{x}}} \mathrm{x}^{\mathrm{i} \varepsilon} \text {. }
$$

The near-tip crack face opening and sliding displacements, $\delta_{2}$ and $\delta_{1}$ (along the negative $\mathrm{x}$ axis), are given by

$$
\delta_{2}+\mathrm{i} \delta_{1}=\frac{1}{2}\left[\frac{\mathrm{C}_{1}+\mathrm{C}_{2}}{(1+2 \mathrm{i} \varepsilon) \cosh (\pi \varepsilon)}\right] \mathrm{K}\left(\frac{|\mathrm{x}|}{2 \pi}\right)^{1 / 2}|\mathrm{x}|^{\mathrm{i} \varepsilon} .
$$

In (1) and (2) the oscillatory exponent, $\varepsilon$, is defined as

$$
\varepsilon=\frac{1}{2 \pi} \ln \left[\frac{1-\beta}{1+\beta}\right]
$$

and for isotropic materials 1 and 2 as designated in Fig. $2, \beta$ is the second Dundurs parameter defined by

$$
\beta=\frac{\mu_{1}\left(\kappa_{2}-1\right)-\mu_{2}\left(\kappa_{1}-1\right)}{\mu_{1}\left(\kappa_{2}+1\right)+\mu_{2}\left(\kappa_{1}+1\right)} .
$$

In $(4) \mu_{j}(j=1,2)$ is the material shear modulus and $\kappa_{j}=\left(3-v_{j}\right) /\left(1+v_{j}\right)(j=1,2)$ for plane stress and $\kappa_{j}=3-4 v_{j}(j=1,2)$ for plane strain. In (2) $C_{j}=\left(\kappa_{j}+1\right) / \mu_{j}(j=1,2)$. In (1) and (2) $\mathrm{K}=\mathrm{K}_{1}+\mathrm{iK} \mathrm{K}_{2}$ is the complex stress intensity factor for interfacial fracture problems, where Arabic subscripts are used to differentiate $K_{1}$ and $K_{2}$ from classically defined stress intensity factors. $K$ takes the dimensional form

$$
\mathrm{K}=\mathrm{K}_{1}+\mathrm{iK} \mathrm{K}_{2}=\mathrm{f} \times \text { (applied stress) } \times\left(\sqrt{\mathrm{h}}^{-\mathrm{i} \varepsilon}\right) \text {, }
$$

where $\mathrm{f}$ is nondimensional and, in general, a complex function of the material properties and the specimen geometry. The parameter $h$ is the dominant length scale or characteristic length of the near-tip problem. For a long delamination crack between plies of equal thickness the characteristic length is the ply thickness. For plies of unequal thickness the characteristic length is the smaller of the two ply thicknesses.

For an interface crack between isotropic layers, the aforementioned oscillatory behavior of the near-tip stresses and displacements is due to the $\mathrm{x}^{\mathrm{i} \varepsilon}$ term in (1) and (2). As $\mathrm{x}$ approaches zero the ratio of $\sigma_{\mathrm{xy}}$ to $\sigma_{\mathrm{yy}}$ or $\delta_{1}$ to $\delta_{2}$ changes rapidly. As a result, the mode of crack extension, which is related to these ratios, is not unique. One approach (which has not yet been discussed) taken to define a mode of crack extension in isotropic interfacial fracture problems involves using the phase angle of the complex $\mathrm{K}$ as a measure of mode. The mode of crack extension is defined by the angle, $\psi$, given by 


$$
\psi=\tan ^{-1}\left[\frac{\operatorname{Im}\left(\mathrm{Kh}^{\mathrm{i} \varepsilon}\right)}{\operatorname{Re}\left(\mathrm{Kh}^{\mathrm{i} \varepsilon}\right)}\right],
$$

where $\psi$ is defined to be independent of the characteristic length, $h$. Inspection of (1) reveals that $\psi$ represents the relative amounts of normal and shear stresses ahead of the crack tip separated from the oscillatory quantity $(\mathrm{x} / \mathrm{h})^{\mathrm{i} \varepsilon}$. This method has been suggested and used by (among others) Rice (1988), Hutchinson, Mear and Rice (1987) and Charalambides, Lund, Evans and McMeeking (1989) as a means for comparing modes of crack extension for isotropic interfacial fracture problems.

\section{Orthotropic Interfacial Fracture}

Consider the interface crack problem illustrated in Figure 2, however materials 1 and 2 are now orthotropic with the $x-y$ plane as a plane of material symmetry. Suo (1990) has formulated equations for the near tip stresses and displacements for such problems. The stress field just ahead of the interfacial crack tip (along the positive $\mathrm{x}$ axis) takes the form

$$
\sigma_{\mathrm{H}} \equiv \sqrt{\frac{\mathrm{H}_{22}}{\mathrm{H}_{11}}} \sigma_{\mathrm{yy}}+\mathrm{i} \sigma_{\mathrm{xy}}=\frac{\left(\mathrm{K}_{1}+\mathrm{i} \mathrm{K}_{2}\right) \mathrm{x}^{\mathrm{i \varepsilon}}}{\sqrt{2 \pi \mathrm{x}}}
$$

The near-tip crack face opening and sliding displacements, $\delta_{2}$ and $\delta_{1}$ (along the negative $\mathrm{x}$ axis), are given by

$$
\delta_{\mathrm{H}} \equiv \sqrt{\frac{\mathrm{H}_{11}}{\mathrm{H}_{22}}} \delta_{2}+\mathrm{i} \delta_{1}=\frac{2 \mathrm{H}_{11}\left(\mathrm{~K}_{1}+\mathrm{iK} \mathrm{K}_{2}\right)|x|^{(1 / 2+i \varepsilon)}}{\sqrt{2 \pi}(1+2 \mathrm{i} \varepsilon) \cosh (\pi \varepsilon)} .
$$

In (7) and (8) the subscript $\mathrm{H}$ in the complex quantities $\sigma_{\mathrm{H}}$ and $\delta_{\mathrm{H}}$ is used to emphasize that the normal stress, $\sigma_{y y}$ and crack face opening displacement, $\delta_{2}$, are multiplied by $\mathrm{H}_{\mathrm{ij}}$ quantities defined below. In (7) and (8) the oscillatory exponent, $\varepsilon$, is given by

$$
\varepsilon=\frac{1}{2 \pi} \ln \left(\frac{1-\beta}{1+\beta}\right),
$$

which is the same form as that used in the isotropic case. For the orthotropic case, the parameter $\beta$ is given by

$$
\beta=\frac{\left[\sqrt{S_{11} S_{22}}+S_{12}\right]_{2}-\left[\sqrt{S_{11} S_{22}}+S_{12}\right]_{1}}{\sqrt{\mathrm{H}_{11} \mathrm{H}_{22}}},
$$

where []$_{2}$ and []$_{1}$ denote the enclosed quantities evaluated for materials 2 and 1 , respectively. The quantities $\mathrm{H}_{11}$ and $\mathrm{H}_{22}$ are functions of the elastic properties of the layers forming the interface and are defined by

$$
\begin{aligned}
& \mathrm{H}_{11}=\left[2 \mathrm{n} \lambda^{1 / 4} \sqrt{S_{11} S_{22}}\right]_{1}+\left[2 n \lambda^{1 / 4} \sqrt{S_{11} S_{22}}\right]_{2} \\
& H_{22}=\left[2 n \lambda^{-1 / 4} \sqrt{S_{11} S_{22}}\right]_{1}+\left[2 n \lambda^{-1 / 4} \sqrt{S_{11} S_{22}}\right]_{2} .
\end{aligned}
$$


Nondimensional parameters used to define $\mathrm{H}_{11}$ and $\mathrm{H}_{22}$ are

$$
\mathrm{n}=\sqrt{\frac{1}{2}(1+\rho)} \quad \lambda=\frac{\mathrm{S}_{11}}{\mathrm{~S}_{22}} \quad \rho=\frac{2 \mathrm{~S}_{12}+\mathrm{S}_{66}}{2 \sqrt{\mathrm{S}_{11} \mathrm{~S}_{22}}} .
$$

Finally, for plane stress problems, the $S_{i j}$ in (10), (11) and (12) are the reduced compliances ( $i$ and $j$ take on values of 1 through 6 ). For plane strain problems, the $S_{i j}$ are replaced by $S_{i j}$ ' defined by

$$
S_{i j}^{\prime}=S_{i j}-\frac{S_{i j} S_{j 3}}{S_{33}} .
$$

The problems associated with oscillating behavior for orthotropic interfacial fracture problems are the same as those associated with the isotropic case. The oscillatory behavior of the near-tip stresses and displacements comes from the $x^{i \varepsilon}$ terms in (7) and (8). Additionally, however, the $H_{\mathrm{ij}}$ quantities in the complex quantities $\sigma_{\mathrm{H}}$ and $\delta_{\mathrm{H}}$ complicate the task of defining a mode for the orthotropic problem. Suo (1990) suggests using the phase of $\mathrm{K}$ as defined for the isotropic case in (6) as a consistent measure of mode for orthotropic interfacial fracture problems. Unfortunately, due to the $\mathrm{H}_{\mathrm{ij}}$ quantity multiplying $\sigma_{22}$ in (7), this mode definition does not have a clear physical significance as is does for the isotropic problem. It also does not simplify to the classical definition of mode for cases where $\varepsilon=0$ but $\mathrm{H}_{11} \neq \mathrm{H}_{22}$. In the next section, a more physically meaningful definition of mode is introduced. As has been done in isotropic interfacial fracture problems, a mode of crack extension is defined that is separated from the non-dimensionalized oscillatory behavior of the solution. However, the mode definition presented in the current study is related to the relative amounts of energy released by an extending crack due to opening vs. that due to sliding. After this mode definition is presented, a method is outlined for extracting it from orthotropic fracture analyses exhibiting oscillatory behavior using the virtual crack closure technique.

\section{Mode Definition for Orthotropic Interfacial Fracture Problems}

For the interface crack geometry shown in Fig. 2, the energy release rate for crack extension in the $\mathrm{x}$ direction can be expressed as

$$
\mathrm{G}_{\text {total }}=\lim _{\Delta \rightarrow 0} \frac{1}{2 \Delta} \int_{0}^{\Delta}\left[\sigma_{22}(\mathrm{x}) \delta_{2}(\Delta-\mathrm{x})+\sigma_{12}(\mathrm{x}) \delta_{1}(\Delta-\mathrm{x})\right] \mathrm{dx},
$$

where the arguments of Irwin (1957) have been used to express the energy per unit width released in propagating the crack a distance $\Delta$ along the $\mathrm{x}$ axis as the energy of the stresses ahead of the tip acting through the displacements behind the tip. Dividing by $\Delta$ and taking the limit as $\Delta$ approaches zero gives the energy release rate. In (14) $G_{\text {total }}$ is the total energy release rate for the extending crack. The first term in (14) is commonly designated as the mode I component of $\mathrm{G}_{\text {total }}$, corresponding to the energy released by normal stresses acting through crack face opening 
displacements. The second term in (14) is commonly designated as the mode II component of $\mathrm{G}_{\text {total }}$, corresponding to the energy released by shear stresses acting through crack face sliding displacements. The oscillatory nature of orthotropic interfacial fracture solutions causes the mode I and mode II components of $\mathrm{G}_{\text {total }}$ to be functions of the crack extension length $\Delta$. No limiting value of crack extension mode is reached in the limit as $\Delta$ approaches zero. Although the individual components of $\mathrm{G}$ are a function of $\Delta, \mathrm{G}_{\text {total }}$ is $\Delta$ independent.

In this section, the explicit oscillatory nature of the mode I and mode II components of $\mathrm{G}_{\text {total }}$ (designated respectively as $\mathrm{G}_{\mathrm{I}}$ and $\mathrm{G}_{\mathrm{II}}$ ) is determined for orthotropic interfacial fracture problems. Some of the procedures used follow those outlined by Raju, Crews and Aminpour (1988) for the isotropic case. Modified energy release rate definitions are then presented that are separated from oscillatory quantities and are thus independent of the virtual crack extension length, $\Delta$. The mode of crack extension based on these definitions thus corresponds to the ratio of energy release due to crack face sliding vs. that due to crack face opening, separated from the oscillatory portion of the solution. As (14) indicates, a definition of mode based on energy release rates involves products of stresses ahead of the crack tip and displacements of the crack faces behind the tip. Mode definitions could also be formulated based individually on the ratio of normal stress vs. shear stress ahead of the crack tip or on the ratio of opening vs. sliding displacements of the crack faces behind the tip. For a crack in a homogeneous material, all three of these mode definitions (based on energy, stress or displacements) are identical. As (7) and (8) show, this is not the case for an interface crack, due to the factor $(1+2 \mathrm{i} \varepsilon)$ in the denominator of (8). This factor causes a small phase shift to exist between the stresses ahead of the tip and the displacements behind the tip.

Although the magnitude of the phase shift between near-tip stresses and displacements is small, its existence requires that some choice be made between the three methods for defining a mode of crack extension. For the orthotropic problem, the use of a mode definition based on energy release rates is preferred because of the $H_{i j}$ factors in the complex quantities $\sigma_{\mathrm{H}}$ and $\delta_{\mathrm{H}}$. The $H_{\mathrm{ij}}$ factors, which cannot be separated from the $\sigma_{\mathrm{H}}$ and $\delta_{\mathrm{H}}$ quantities, cancel in the calculation of energy release rates. As a result, a mode definition based on energy release rates simplifies to the classical definition of mode for cases where $\varepsilon=0$ but $\mathrm{H}_{11} \neq \mathrm{H}_{22}$.

Because the oscillatory behavior of $\sigma_{\mathrm{H}}$ and $\delta_{\mathrm{H}}$ are known, the initial step in determining the oscillatory behavior of $\mathrm{G}_{\mathrm{I}}$ and $\mathrm{G}_{\mathrm{I}}$ is to express them in terms of $\sigma_{\mathrm{H}}$ and $\delta_{\mathrm{H}}$. First, define two real functions $\Phi_{1}$ and $\Phi_{2}$ in terms of $\sigma_{\mathrm{H}}$ and $\delta_{\mathrm{H}}$

$$
\begin{aligned}
& \Phi_{1} \equiv \operatorname{Re}\left[\int_{0}^{\Delta} \sigma_{\mathrm{H}}(\mathrm{x}) \overline{\delta_{\mathrm{H}}(\Delta-\mathrm{x})} \mathrm{dx}\right] \\
& \Phi_{2} \equiv \operatorname{Re}\left[\int_{0}^{\Delta} \sigma_{\mathrm{H}}(\mathrm{x}) \delta_{\mathrm{H}}(\Delta-\mathrm{x}) \mathrm{dx}\right],
\end{aligned}
$$


where the overbar designates the complex conjugate of the quantity below it. Using the energy release rate definitions given in (14), $\mathrm{G}_{I}, \mathrm{G}_{\mathrm{II}}$ and $\mathrm{G}_{\text {total }}$ can be expressed in terms of $\Phi_{1}$ and $\Phi_{2}$ as follows

$$
\begin{gathered}
\mathrm{G}_{\mathrm{I}}=\lim _{\Delta \rightarrow 0} \frac{1}{4 \Delta}\left[\Phi_{1}+\Phi_{2}\right] \\
\mathrm{G}_{\mathrm{II}}=\lim _{\Delta \rightarrow 0} \frac{1}{4 \Delta}\left[\Phi_{1}-\Phi_{2}\right] \\
\mathrm{G}_{\text {total }}=\mathrm{G}_{\mathrm{I}}+\mathrm{G}_{\mathrm{II}}=\lim _{\Delta \rightarrow 0} \frac{1}{2 \Delta}\left[\Phi_{1}\right] .
\end{gathered}
$$

Through the definitions for $\Phi_{1}$ and $\Phi_{2}$ the energy release rates have now been expressed in terms of $\sigma_{\mathrm{H}}$ and $\delta_{\mathrm{H}}$. The oscillatory behavior of $\Phi_{1}$ and $\Phi_{2}$ can be determined by substituting the asymptotic stress and displacement equations (7) and (8) into (15) and integrating. The forms of $\Phi_{1}$ and $\Phi_{2}$ to be integrated are

$$
\begin{gathered}
\Phi_{1}=\frac{\mathrm{H}_{11}}{\pi \cosh \pi \varepsilon} \mathrm{K} \overline{\mathrm{K}} \operatorname{Re}\left[\frac{1}{1-2 \mathrm{i} \varepsilon} \int_{0}^{\Delta}\left(\frac{\Delta-\mathrm{x}}{\mathrm{x}}\right)^{1 / 2-\mathrm{i} \varepsilon} \mathrm{dx}\right] \\
\Phi_{2}=\frac{\mathrm{H}_{11}}{\pi \cosh \pi \varepsilon} \operatorname{Re}\left[\frac{\mathrm{K}^{2}}{1+2 \mathrm{i} \varepsilon} \int_{0}^{\Delta}\left(\frac{\Delta-\mathrm{x}}{\mathrm{x}}\right)^{1 / 2}((\Delta-\mathrm{x}) \mathrm{x})^{\mathrm{i} \varepsilon} \mathrm{dx}\right] .
\end{gathered}
$$

Use of the trigonometric substitution $x=\Delta \sin ^{2}(\beta)$ in the integral in the expression for $\Phi_{1}$ gives an expression in terms of the Beta function. Identities between the Beta and Gamma functions allow this expression to be simplified so that $\Phi_{1}$ is given by

$$
\Phi_{1}=\frac{\mathrm{H}_{11}}{2 \cosh ^{2} \pi \varepsilon} \mathrm{K} \overline{\mathrm{K}} \Delta,
$$

which is non-oscillatory. Use of the same trigonometric substitution in the integral contained in $\Phi_{2}$ also results in an expression in terms of the Beta function. The expression for $\Phi_{2}$ cannot be simplified in the way that $\Phi_{1}$ can. The function $\Phi_{2}$ is thus given by

$$
\Phi_{2}=\frac{2 \Delta \mathrm{H}_{11}}{\pi \cosh \pi \varepsilon} \operatorname{Re}\left[\frac{\left(\mathrm{Kh}^{\mathrm{i} \varepsilon}\right)^{2}}{1+2 \mathrm{i} \varepsilon}\left(\frac{\Delta}{\mathrm{h}}\right)^{2 \mathrm{i} \varepsilon} \int_{0}^{\pi / 2} \cos ^{2}(\beta)(\cos (\beta) \sin (\beta))^{2 \mathrm{i} \varepsilon} \mathrm{d} \beta\right],
$$

where the integral is equivalent to $B(z, w)$ with $z=1 / 2+i \varepsilon$ and $w=3 / 2+i \varepsilon$. In (19) the crack extension length, $\Delta$, has been normalized by the characteristic length, $h$. With respect to the near-crack-tip fields, $h$ is the dominant length scale determining the size of the region in which the near-tip oscillations dominate. For the case of a long delamination crack, the layer thickness (or the smallest layer thickness if the layers forming the interface have unequal thicknesses) is the characteristic length.

By determining the oscillatory behavior of $\Phi_{2}$, the oscillatory behaviors of the energy release rate quantities for orthotropic interfacial fracture problems have been isolated. The oscillatory behavior of $\Phi_{2}$ can be eliminated by defining a $\Phi_{2}$ ' as 


$$
\Phi_{2}{ }^{\prime} \equiv \operatorname{Re}\left[\left(\frac{\Delta}{h}\right)^{-2 i \varepsilon} \cdot \int_{0}^{\Delta} \sigma_{H}(x) \delta_{H}(\Delta-x) d x\right],
$$

which is $\Phi_{2}$ with the oscillatory quantity $(\Delta / \mathrm{h})^{2 \mathrm{i} \varepsilon}$ extracted. Now define $\mathrm{G}_{1}, \mathrm{G}_{2}$ (designated with Arabic subscripts to differentiate them from $\mathrm{G}_{\mathrm{I}}$ and $\mathrm{G}_{\mathrm{II}}$ in (16)) and $\mathrm{G}_{\text {total }}$ as

$$
\begin{aligned}
G_{1} & =\lim _{\Delta \rightarrow 0} \frac{1}{4 \Delta}\left[\Phi_{1}+\Phi_{2}{ }^{\prime}\right] \\
G_{2} & =\lim _{\Delta \rightarrow 0} \frac{1}{4 \Delta}\left[\Phi_{1}-\Phi_{2}{ }^{\prime}\right] \\
G_{\text {total }} & =G_{1}+G_{2}=\lim _{\Delta \rightarrow 0} \frac{1}{2 \Delta}\left[\Phi_{1}\right] .
\end{aligned}
$$

In these modified energy release rate definitions, $G_{\text {total }}$ is still $\Delta$ independent, however, the $G_{1}$ and $G_{2}$ portions are also $\Delta$ independent. The relations given in (20) and (21) define energy release rate quantities that are independent of the crack extension length, $\Delta$, for orthotropic material oscillatory interfacial fracture models. These relations have been derived by first isolating the $(\Delta / \mathrm{h})^{2 \mathrm{i} \varepsilon}$ oscillatory behavior of the quantity $\Phi_{2}$ and then defining energy release rate components in terms of a quantity $\Phi_{2}$ ' that has this oscillatory quantity extracted from it. The result is mode I and mode II energy release rate components that are separated from the oscillatory behavior of the solution.

The definitions for individual mode components derived in this section are analogous to the mode definition discussed in the section on isotropic interfacial fracture problems (see the discussion related to (6)). In that case, the mode of crack extension was related to the ratio of normal stresses to shear stresses ahead of the crack tip, separated from the oscillatory quantity $(\mathrm{r} / \mathrm{h})^{\mathrm{i} \varepsilon}$. The same approach is used in deriving (20) and (21), except that mode components are given in terms of energy release rates. A phase angle, $\psi$, analogous to that defined in (6) can be used to designate mode mix,

$$
\psi=\tan ^{-1}\left[\sqrt{\frac{\mathrm{G}_{2}}{\mathrm{G}_{1}}}\right]
$$

where $G_{1}$ and $G_{2}$ are given by (21).

The basis for the mode definitions derived in this section is that the near-tip oscillatory behavior of interfacial fracture solutions is non-physical. It is therefore argued that a mode of crack extension defined to be separated from oscillatory quantities in the solution can serve as a reasonable and consistent definition for comparing modes between problems. The normalization of $\Delta$ by $h$ used in (20) is chosen because $h$ is the length scale determining the size of the region in which the near-tip oscillations dominate. It is important to note, however, that this normalization is not the only one that could be reasonably argued. For example, one might argue that the zone dominated by the near-crack-tip stresses and displacements (often referred to as the $\mathrm{K}$ field) will 
extend to a distance that is a fraction of $h$. Therefore, a normalization of $\Delta$ by some fraction of $h$ in (20) could be proposed. The validity of any proposed normalization (including the one proposed in this paper) must eventually be proven by its ability to consistently relate interfacial fracture resistances. It is important to recognize, however, that the numerical difference in the mode components that would result from any physically reasonable change in normalization would be small. Because the magnitude of $\varepsilon$ is very small for most physical problems (see Suo (1990)), the mode change from oscillations over a range of distances from the crack tip of $h$ to $\mathrm{h} / 20$ is very small. From a practical standpoint, determination of which normalization is the "correct" one would be difficult and would likely have minimal bearing on the physical task of consistently comparing modes of crack extension between fracture problems.

\section{Virtual Crack Closure Technique}

The virtual crack closure technique (VCCT) is a method used to extract mode I and mode II energy release rate components from numerical (typically finite element) analyses. The method, which is detailed by Rybicki and Kanninen (1977), is based on the Irwin representation of energy release rate for self-similar crack extension given in (14). The method involves expressing the mode I and mode II portions of $\mathrm{G}_{\text {total }}$ (see (14)) in terms of nodal forces ahead of the crack tip and nodal displacements behind the tip. This can be represented by the expressions

$$
\mathrm{G}_{\mathrm{I}}=\frac{1}{2 \Delta} \sum_{\mathrm{j} \text { nodes }} \mathrm{F}_{22_{j}} \delta_{2_{j}} \quad \mathrm{G}_{\mathrm{II}}=\frac{1}{2 \Delta} \sum_{\text {nndes }} \mathrm{F}_{12_{j}} \delta_{1_{j}},
$$

where $F_{22 j}$ and $F_{12 j}$ are, respectively, the normal and shear nodal forces per unit thickness over distance $\Delta$ ahead of crack tip and $\delta_{2 j}$ and $\delta_{1 \mathrm{j}}$ are "corresponding" nodal crack opening and sliding displacements over distance $\Delta$ behind the crack tip. Corresponding nodes are designated in Figure 3. In the VCCT, $\Delta$ is referred to as the virtual crack extension length. The VCCT is applied by using (23) to calculate energy release rates for smaller and smaller values of $\Delta$, until no significant changes in $\mathrm{G}$ values occur. In applying the VCCT to oscillatory interface crack solutions, only $\mathrm{G}_{\text {total }}$ reaches a limiting value as $\Delta$ is decreased. The relations in (23) assume that nodes are equally spaced ahead of and behind the crack tip, as would be the case if non-singular elements are used in the analysis. The technique can also be used with singular crack tip elements using relations given in Raju, Crews and Aminpour (1988).

\section{Modified Virtual Crack Closure Technique}

In this section, a modified VCCT is formulated to allow extraction of the energy release rates defined in (21) from a numerical model that exhibits oscillatory behavior. To do this, the energy release rate quantities in (21) are equivalently expressed in terms of nodal forces and displacements. Define 


$$
\begin{aligned}
\mathrm{F}_{\mathrm{H}_{\mathrm{J}}} & \equiv \sqrt{\frac{\mathrm{H}_{22}}{\mathrm{H}_{11}}} \mathrm{~F}_{22,}+\mathrm{iF}_{12 \mathrm{j}} \\
\delta_{\mathrm{H}_{\mathrm{J}}} & \equiv \sqrt{\frac{\mathrm{H}_{11}}{\mathrm{H}_{22}}} \delta_{2,}+\mathrm{i} \delta_{1_{\mathrm{j}}},
\end{aligned}
$$

where $F_{22 j}$ and $F_{12 j}$ and $\delta_{2 j}$ and $\delta_{1 j}$ are defined with respect to Fig. 3 as they are for the traditional VCCT. With these complex quantities defined, $\Phi_{1}$ and $\Phi_{2}$ ' can be expressed as

$$
\begin{gathered}
\Phi_{1}=\operatorname{Re}\left[\sum_{j \text { nodes }} F_{\mathrm{H}_{j}} \overline{\delta_{\mathrm{H}_{j}}}\right] . \\
\Phi_{2}{ }^{\prime}=\operatorname{Re}\left[\left(\frac{\Delta}{\mathrm{h}}\right)^{-2 i \varepsilon} \sum_{j \text { nodes }} \mathrm{F}_{\mathrm{H}_{j}} \delta_{\mathrm{H}_{j}}\right]
\end{gathered}
$$

and the energy release rate $\left(G_{1}, G_{2}\right.$ and $\left.G_{\text {total }}\right)$ definitions in terms of $\Phi_{1}$ and $\Phi_{2}$ ' are simply those given in (21).

In applying this modified virtual crack closure technique, the same quantities (nodal forces ahead of tip and nodal displacements behind the tip) are used as in the traditional VCCT. The quantities are simply re-packaged in the complex forms given in (24). The only additional effort required is that needed to calculate the quantities $\mathrm{H}_{11}, \mathrm{H}_{22}$ and $\varepsilon$ (defined in (9) through (13)) and to perform the complex multiplication indicated in (25). Use of the modified VCCT outlined in this section is similar to the existing approach of using the traditional VCCT with a virtual crack extension length, $\Delta$, that is large compared to the zone dominated by $\varepsilon$ effects (this approach was described in the final portion of the Introduction). The goal of using the traditional VCCT with a large value of $\Delta$ is to avoid oscillatory effects by simply avoiding the near-tip region. The advantage of this modified VCCT is that small values of $\Delta$ can be used while still avoiding oscillatory effects because the oscillatory effects are extracted analytically. This allows limiting values of energy release rates to be obtained as $\Delta$ approaches zero.

A relationship exists between the modes of crack extension obtained using the traditional and modified VCCT. The mode of crack extension obtained using the modified technique corresponds to the mode one would obtain using $\Delta=\mathrm{h}$ in the traditional VCCT, if the zone dominated by the near-crack-tip fields extended to a distance $h$ from the crack tip. This relationship is due to the normalization with respect to $h$ used in the modified VCCT (see (19) and (25)). Because a second interface exists at a distance $h$ from the crack tip, in practical problems the near-tip zone will not extend to a distance $\Delta=\mathrm{h}$. As a result, direct comparison of the two methods based on their predictions for $\Delta=\mathrm{h}$ is generally not possible.

\section{Application}

In the previous section, a modified VCCT has been formulated to extract the energy release rate quantities defined in (21). In theory, application of this modified VCCT to numerical 
solutions will result in $\Delta$-independent modes of crack extension. This section demonstrates how the methods outlined in this study perform in practice. Results of application of the modified VCCT to finite element models of two orthotropic interfacial fracture problems are presented. An example of the finite element mesh used in this study is shown in Figure 4. The model is constructed out of eight-noded plane strain quadrilateral interpolation elements using the finite element package ABAQUS. Refined singular and nonsingular element meshes have been used near the crack tip. For the nonsingular near-tip mesh, nodes are located at equally spaced $r$ intervals from the crack tip. For the singular near-tip mesh, the first elements surrounding the tip are quarter-point elements to capture the $1 / \sqrt{ }$ r near-tip strain dependence. Subsequent elements are biased toward the crack tip to give higher element resolution there. The density of the neartip mesh was varied to check for convergence; however, for the results presented here, the singular and non-singular near-tip meshes consist of 18 rings of elements meshed over a length equal to $h / 2$, where $h$ is the smaller thickness of the two plies forming the interface.

Two problems were analyzed to evaluate the methods proposed in the previous sections. The problems analyzed are shown in Figure 5 and are designated as test case \#1 and test case \#2. The two test cases consist of plane strain drop-ply configurations of $0^{\circ}$ and $90^{\circ} 0.005$ inch thick graphite-epoxy plies. Each test problem has displacements constrained to equal zero on the left edge (built-in conditions) and a shear load distributed along the right edge. Test case \#1 has been previously modeled in work by Wang, et al (1993) on skin-stiffener debond modeling using plate elements. Test case \#2 was chosen to allow demonstration of the behavior of the modified VCCT for both positive and negative values of $\varepsilon$. Unidirectional ply properties used in the analysis are identical to those used by Wang, et al (1993) and are given in Table 1. Although the magnitude of $\varepsilon$ is the same for both cases, the physical behavior of the problems is very different. Test case \#2 exhibits more compliant response compared to test case \#1. The energy release rates for test case \#2 are much higher than those for test case \#1 and the mode of crack extension for the two problems is very different.

Figures 6 and 7 give plots of the ratios $G_{I I} / G_{I}$ and $G_{2} / G_{1}$ as a function of $\Delta / h$ for test case $\# 1$ and test case \#2, respectively. In both figures, the ratio is plotted as calculated using the modified and traditional virtual crack closure techniques. In Figure 7, the two points to the far left represent values calculated using each virtual crack closure technique over the first element of a mesh containing singular crack tip elements. The four pairs of points to the right represent values calculated using one, two, three, and four elements from the crack tip of a mesh containing non-singular crack-tip elements. The plot in Figure 7 contains only points from a model with non-singular crack tip elements.

Figures 6 and 7 show that application of a traditional VCCT to these two cases using small values of $\Delta / \mathrm{h}$ results in significant changes in $\mathrm{G}_{\mathrm{II}} / \mathrm{G}_{\mathrm{I}}$ with $\Delta$. The positive value of $\varepsilon$ in test 
case \#1 causes $G_{I I} / G_{I}$ to decrease with decreases in $\Delta$ in Fig. 6 . In Fig. 7 , the negative value of $\varepsilon$ in test case $\# 2$ causes $\mathrm{G}_{\mathrm{II}} / \mathrm{G}_{\mathrm{I}}$ to increase with decreases in $\Delta$. As Figures 6 and 7 indicate, use of the modified VCCT results in $G_{2} / G_{1}$ ratios that are essentially independent of $\Delta$. Thus the modified VCCT appears to work well in allowing extraction of a consistent mode of crack extension from an oscillatory solution. Slight variations in the $\mathrm{G}_{2} / \mathrm{G}_{1}$ values obtained using only the first elements from the crack tip can be seen. These can be attributed to the fact that rapid changes in displacements as the crack tip is approached can cause the first element from the tip to give less accurate nodal force and displacement results than subsequent elements away from it. As mentioned in the previous section, the mode of crack extension extracted using the modified VCCT corresponds to that from a traditional VCCT using $\Delta=h$. The results shown in Figs. 6 and 7 , which show increasing agreement between the two methods as $\Delta$ is increased, qualitatively agree with this. Curves for the two methods would continue to approach each other if $\Delta$ were increased further, as long as the length $\Delta$ remained within the region dominated by the near-crack tip fields. Again, it should be emphasized actual application of the traditional VCCT for values of $\Delta$ approaching $\mathrm{h}$ would generally give incorrect results because the near-tip fields no longer dominate at a distance $h$ from the crack tip.

The results in Figs. 6 and 7 point out one final advantage of the modified VCCT, related to the task of verifying the convergence of a finite element solution. By looking at the modified VCCT results in Figs. 6 and 7, one can be reasonably well-assured that the near-tip mesh is sufficiently refined. Because the ratio of $\mathrm{G}_{2} / \mathrm{G}_{1}$ is constant for the modified VCCT applied over a number of elements, it is clear that multiple elements are within the region dominated by the near-crack-tip fields. Also, the near-tip elements are correctly picking up the variations in mode due to the oscillatory nature of the solution (because when the modified VCCT factors out oscillatory quantities a constant value results). Thus the modified VCCT can aid numerical modelers in determining if they have a sufficiently refined near-tip mesh, substantially reducing the need to compare results from meshes with many levels of refinement to explicitly verify convergence.

\section{Conclusions}

In this study, the problem of extracting consistent modes of crack extension from oscillatory near-crack-tip solutions has been addressed. In particular, a mode definition for the problem of a crack along an interface between two in-plane orthotropic layers has been developed and applied within a modified version of the virtual crack closure technique. Knowledge of the near-tip oscillatory stress and displacement fields for an interface crack between two in-plane orthotropic materials has been used to determine the explicit oscillatory nature of mode I and mode $I I$ energy release rate components. Energy release rates have 
subsequently been defined that are separated from oscillatory quantities in the solution. These energy release rate definitions have been used to formulate a modified Virtual Crack Closure Technique (VCCT) that allows extraction of crack extension modes that are $\Delta$ independent. The modified VCCT uses the same quantities (near crack tip nodal displacements and forces) extracted from a finite element analysis using the traditional VCCT. The modified technique has been applied successfully to two numerical test cases.

The methods developed in this study can be applied to any in-plane orthotropic debonding problem, however the most obvious application is to composite delamination problems. The current work is directly applicable to planar composite delamination problems where the plies or sublaminates are modeled as in-plane orthotropic layers. The definition for mode of crack extension defined in this study has potential as a consistent mode definition to be used in such problems. Additionally, the ability to extract $\Delta$-independent mode values using a virtual crack closure technique can aid numerical modelers in determining solution convergence.

\section{Acknowledgment}

The author gratefully acknowledges the support of the NASA Langley Mechanics of Materials Branch and the American Society for Engineering Education through their joint Summer Faculty Fellowship Program. The author would also like to thank Kevin O'Brien for his thoughts and insight related to this research.

\section{References}

Atkinson, C. (1977) On stress singularities and interfaces in linear elastic fracture mechanics. International Journal of Fracture, 13, 807-820

Chai, H. (1992) Experimental evaluation of mixed-mode fracture in adhesive bonds. Experimental Mechanics, 32, 296-303.

Charalambides, P.G., Lund, J., Evans, A.G. and McMeeking, R.M. (1989) A test specimen for determining the fracture resistance of bimaterial interfaces. Journal of Applied Mechanics, 56, 77-82.

Davidson, B.D. (1993) Prediction of energy release rate for edge delamination using a crack tip element. Proc. Fifth ASTM Symposium on Composite Materials: Fatigue and Fracture, Atlanta, May 4-6.

He, M.Y., and Hutchinson, J.W. (1989) Kinking of a crack out of an interface. Journal of Applied Mechanics, 56, 270-278.

Hutchinson, J.W., Mear, M.E. and Rice, J.R. (1987) Crack paralleling an interface between dissimilar materials. Journal of Applied Mechanics, 53, 828-832. 
Irwin, G.R. (1957) Analysis of stresses and strains near the end of a crack traversing a plate. Journal of Applied Mechanics, 24, 361-364.

O'Brien, T.K. (1982) Characterization of delamination onset and growth in a composite laminate. Damage in Composite Materials, ASTM STP 775, 140-147.

Raju, I.S., Crews, J.H., Jr. and Aminpour, M.A. (1988) Convergence of strain energy release rate components for edge-delaminated composite laminates. Engineering Fracture Mechanics, 30, 383-396.

Reeder, J.R. and Crews, J.H., Jr. (1990) Mixed-mode bending method for delamination testing. AIAA Journal, 28, 1270-1276.

Rice, J.R. (1988) Elastic fracture mechanics concepts for interfacial cracks. Journal of Applied Mechanics, 55, 98-103.

Rybicki E.F. and Kanninen M. F. (1977) A finite element calculation of stress intensity factors by modified crack closure integral. Engineering Fracture Mechanics, 9, 931-938.

Suo, Z. (1990) Singularities, interfaces and cracks in dissimilar anisotropic media. Proceedings of the Royal Society of London, A 427, 331-358.

Suo, Z. and Hutchinson, J.W. (1989) Sandwich test specimens for measuring interface crack toughness. Materials Science and Engineering, A107, 135-143.

Suo, Z. and Hutchinson, J.W. (1990) Interface crack between two elastic layers. International Journal of Fracture, 43, 1-18.

Wang, A.S.D. and Crossman, F.W. (1980) Initiation and growth of transverse cracks and edge delamination in composite laminates parts 1 and 2. Journal of Composite Materials, supplemental volume, 71-106.

Wang, J.T., Raju, I.S., Davila, C.G. and Sleight, D.W. (1993) Computation of strain energy release rates for skin-stiffener debonds modeled with plate elements. Proc. 34th AIAA/ASME/ASCE/AHS/ASC Structures, Structural Dynamics and Materials Conference, Part 3, Paper AIAA 93-1501-CP, 1680-1692.

Wang, S.S. (1982) Fracture mechanics for delamination problems in composite materials. Progress in Science and Engineering of Composites, T. Hayashi, K. Kawata, and S. Umekawa, Ed., Proc. ICCM-IV, Tokyo, 287-296. 


\section{Table 1}

Graphite-Epoxy Ply Properties Used in the Numerical Test Cases

(All moduli are in psi)

\begin{tabular}{ll}
\hline$E_{11}=19.5 \times 10^{6}$ & $E_{22}=E_{33}=1.48 \times 10^{6}$ \\
$\mu_{12}=\mu_{13}=0.80 \times 10^{6}$ & $\mu_{23}=0.497 \times 10^{6}$ \\
$v_{12}=v_{13}=0.30$ & $v_{23}=0.49$ \\
\hline
\end{tabular}

Note: 1 designates the direction along the fiber, 2 designates the in-plane direction normal to the fiber and 3 designates the out-of-plane direction normal to the fiber.

These properties are the same as those used by Wang, et al (1993). 

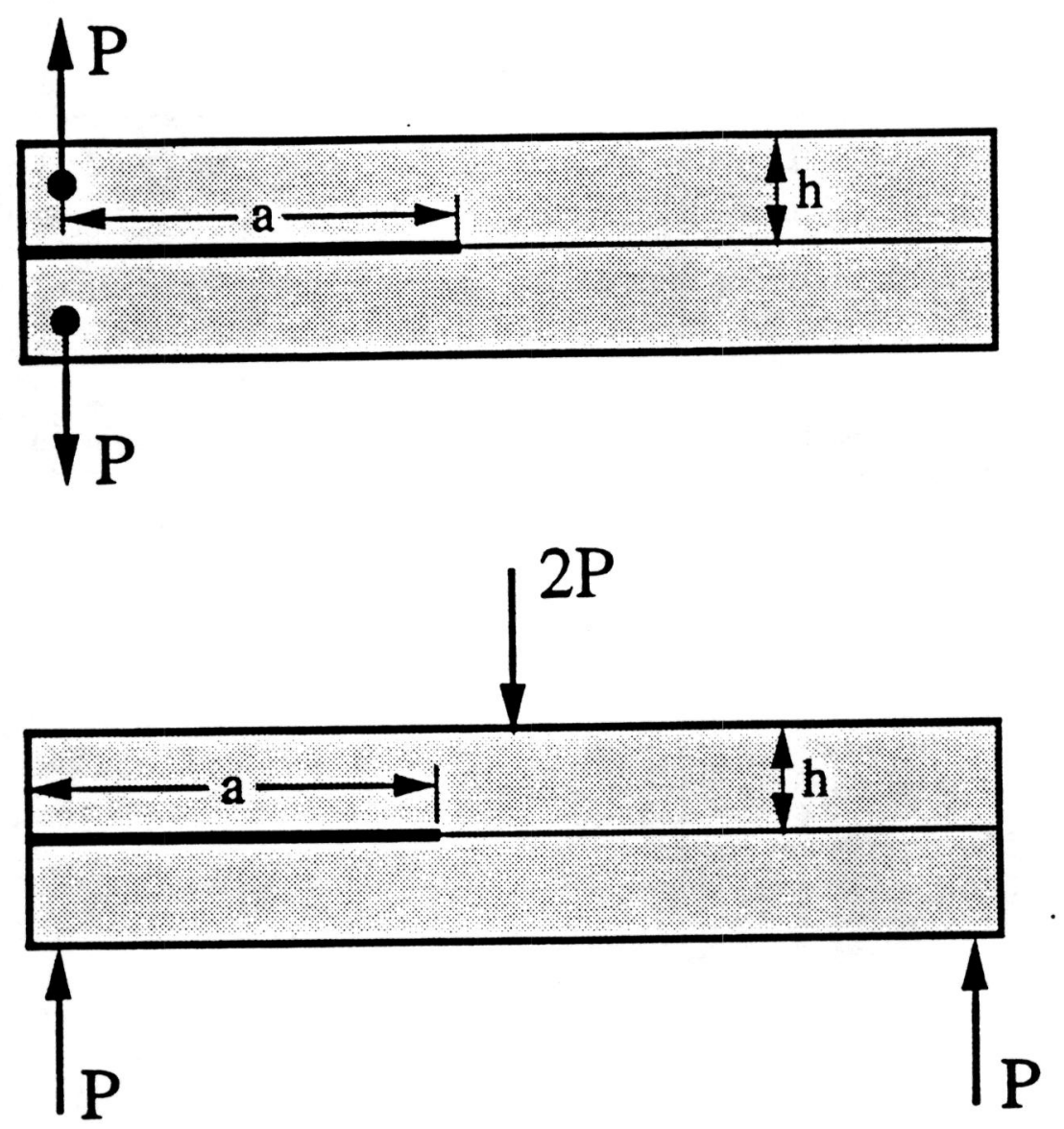

Figure 1 Double Cantilever Beam and Edge Notched Flexure Fracture Toughness Specimens 


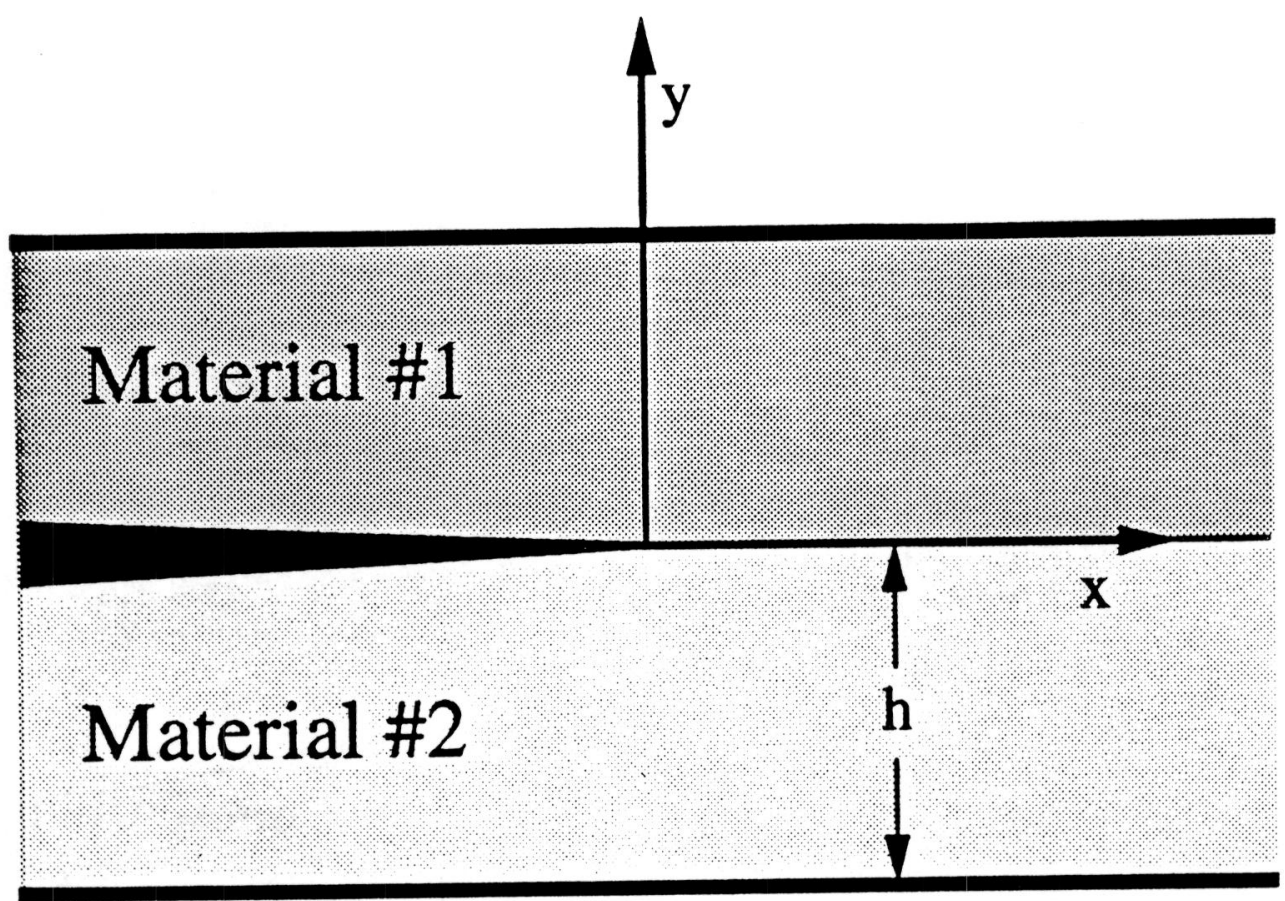

Figure 2 Interface Crack Between Two Elastic Layers 


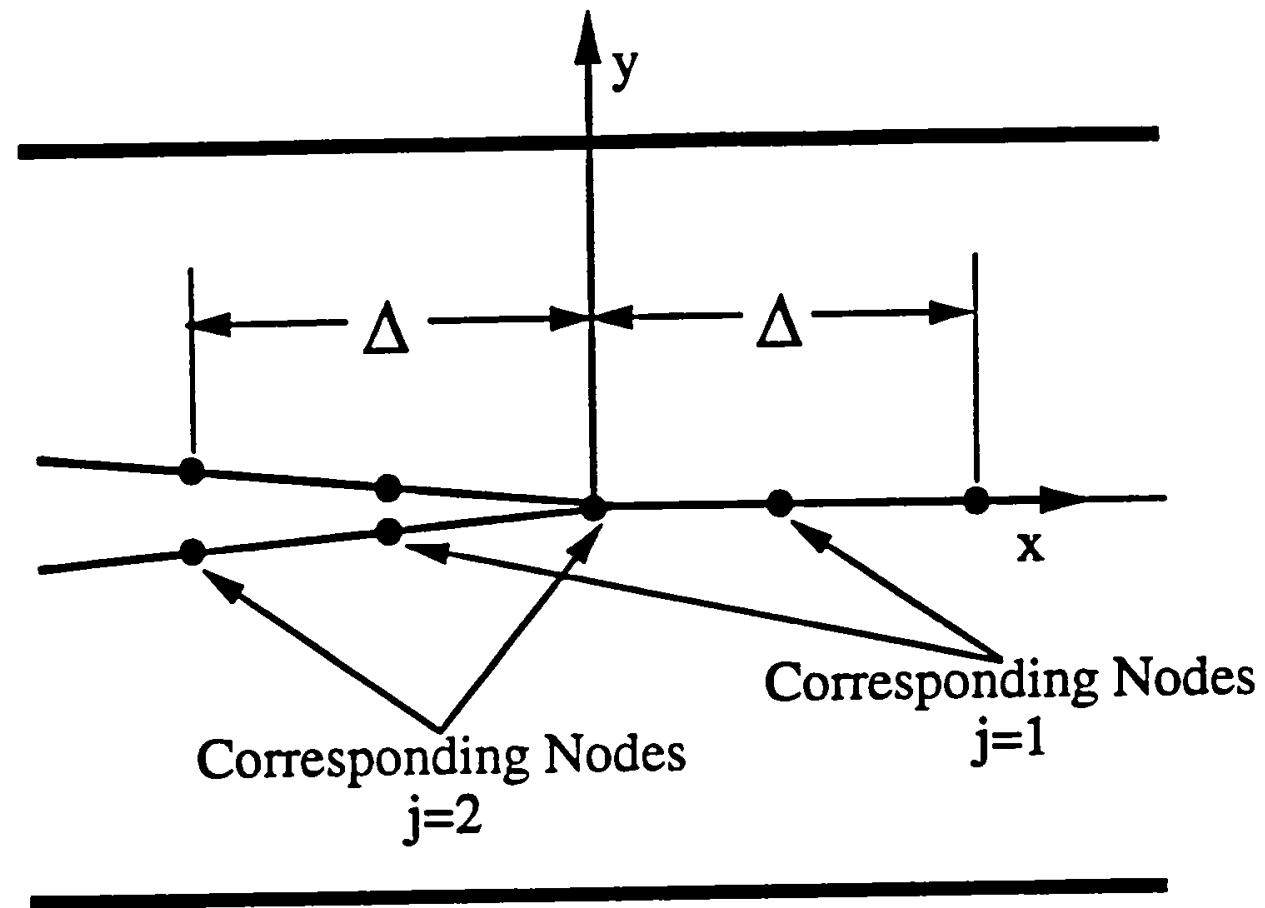

Figure 3 Virtual Crack Closure Technique Parameters 

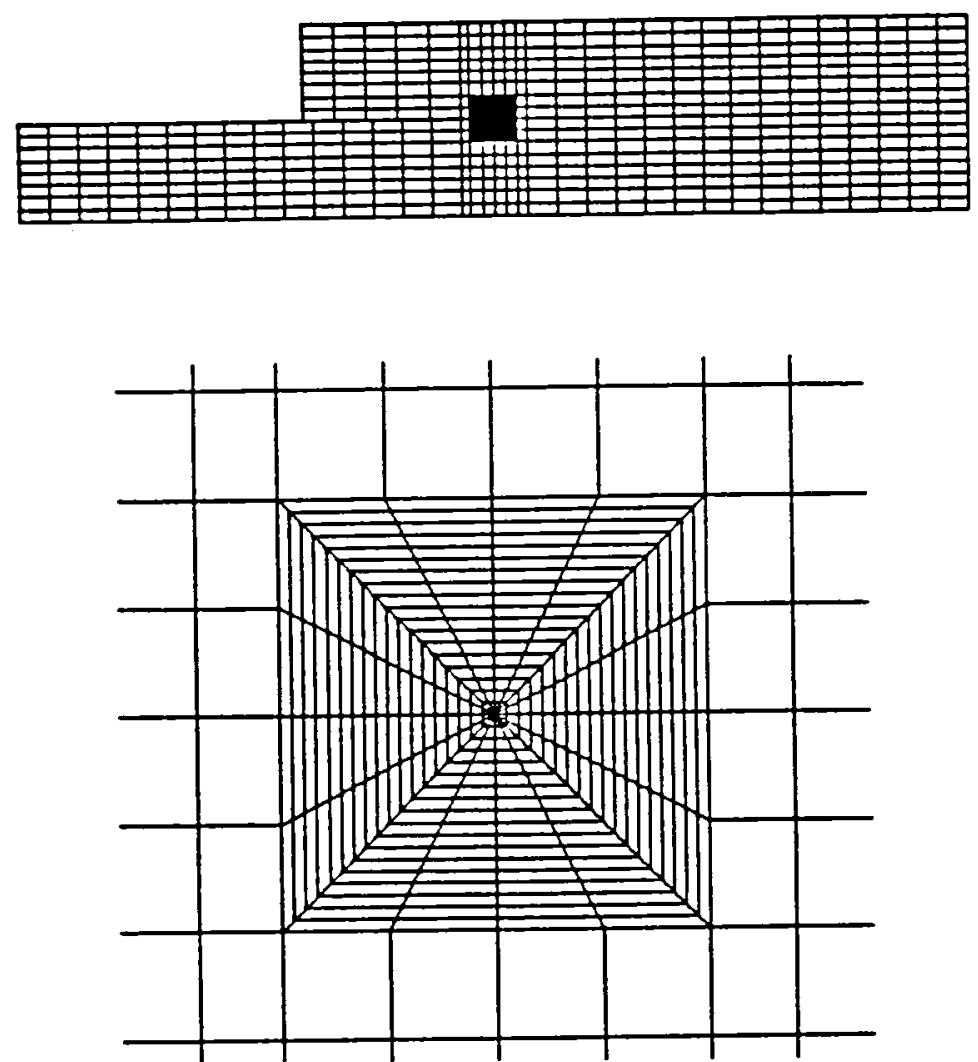

Figure 4 Finite Element Mesh with Non-Singular Crack Tip Elements 

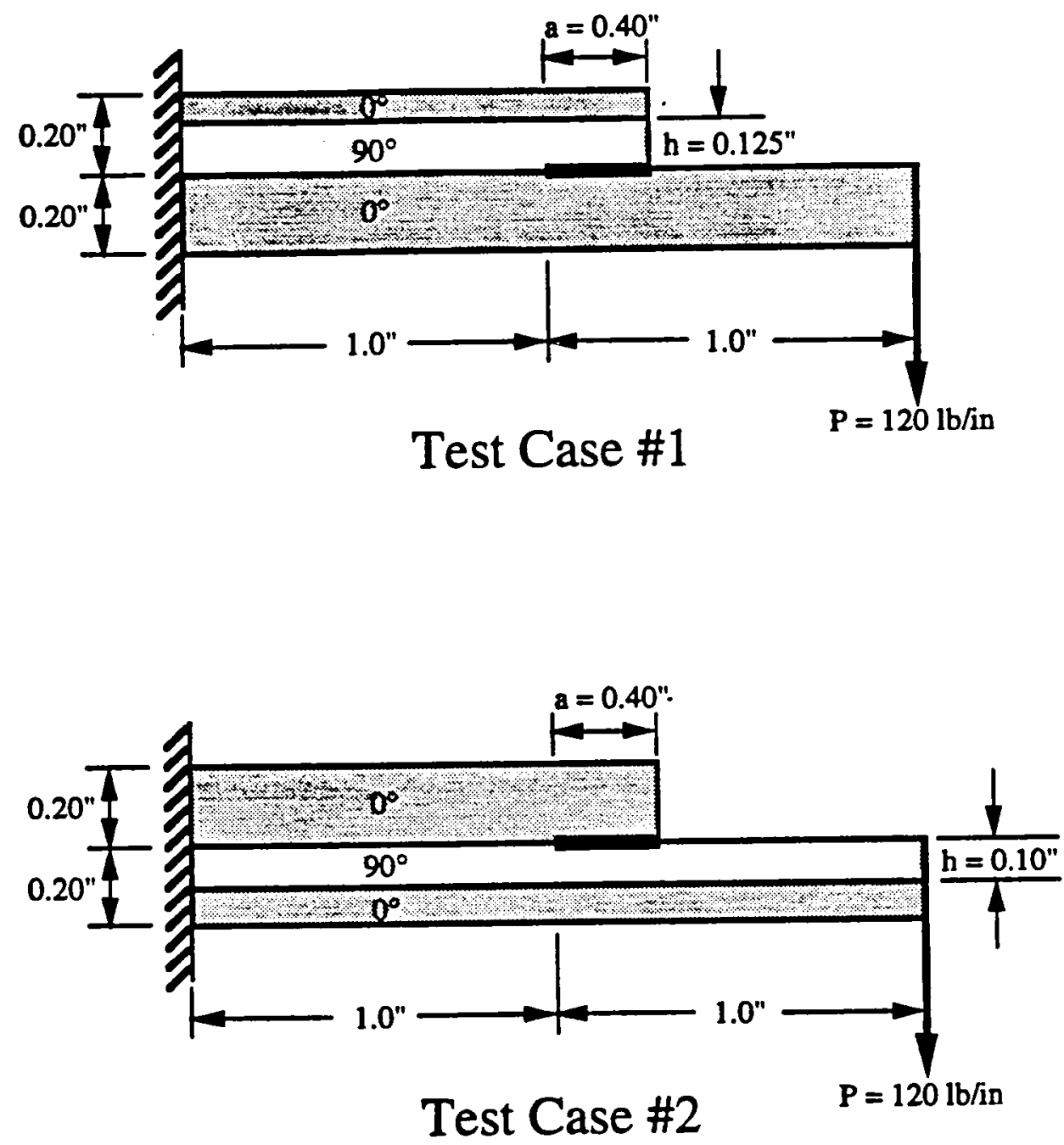

Figure 5 Diagrams of Test Case \#1 and Test Case \#2 


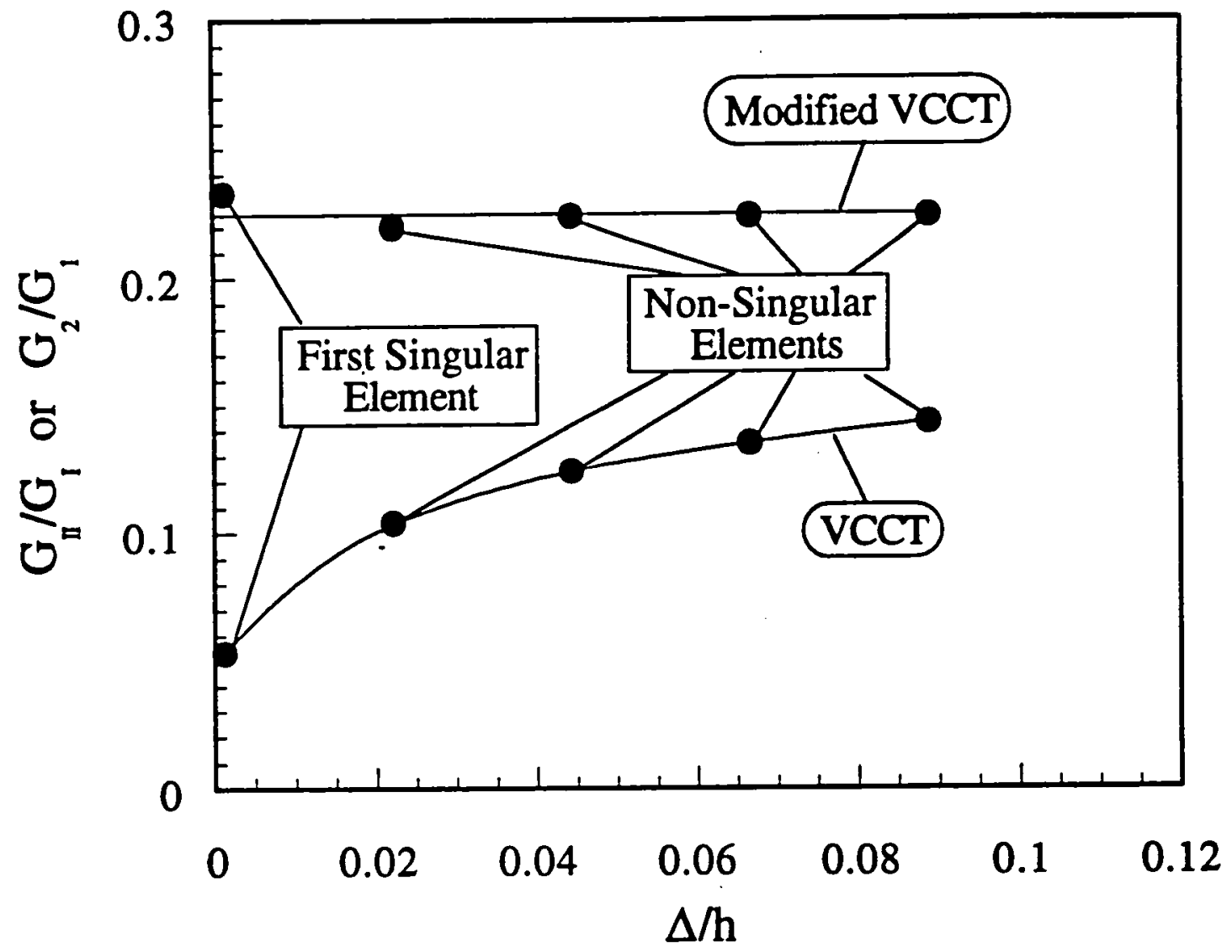

Figure 6 Energy Release Rate Ratios vs. Normalized Virtual Crack Extension Length, $\Delta$, for Test Case \#1 


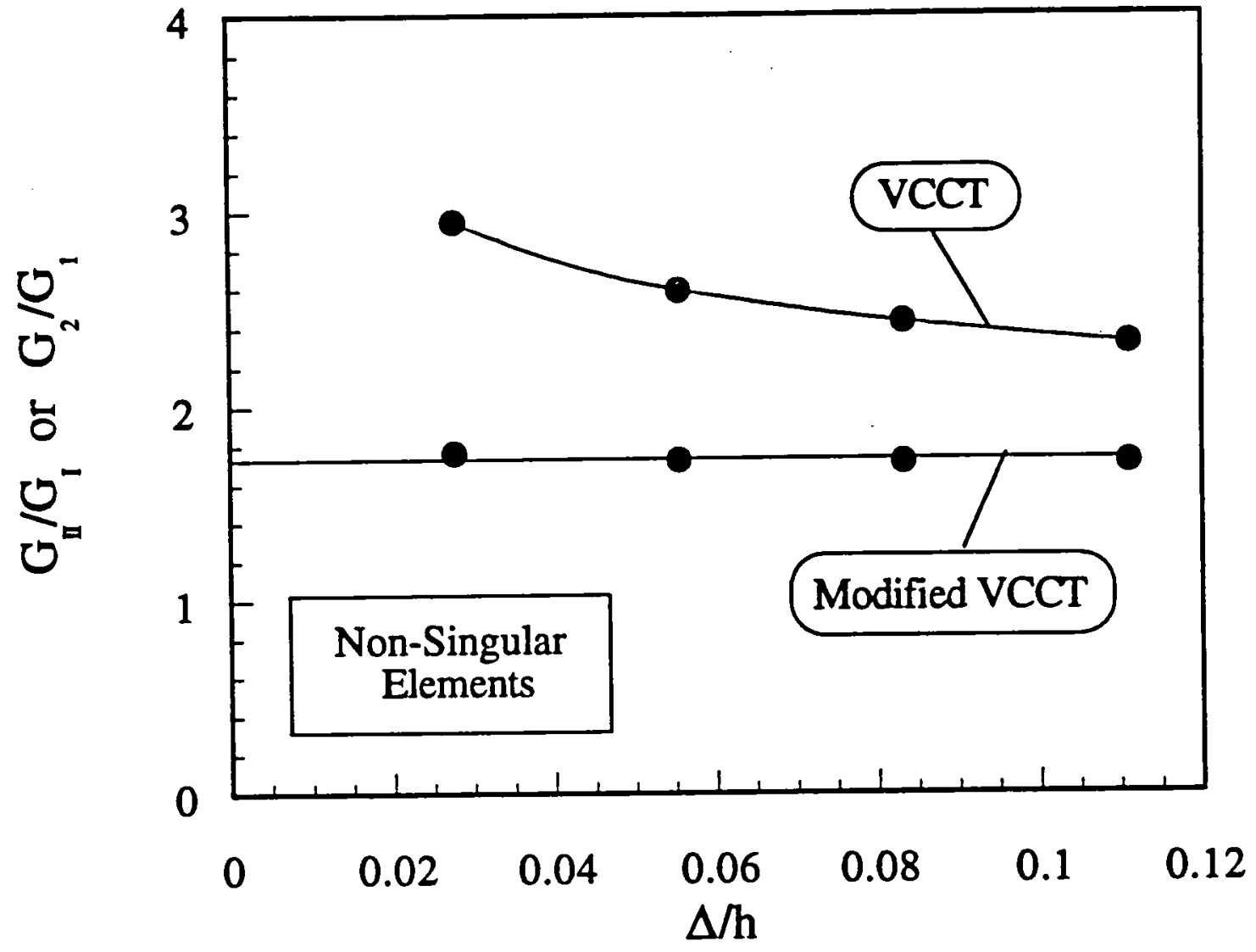

Figure 7 Energy Release Rate Ratios vs. Normalized Virtual Crack Extension Length, $\Delta$, for Test Case \#2 

Public reporting burden for this collection of information is estimated to average i hour per response, including the time for reviewing instructions, searching existing data sources. gathering and maintaining the data needed, and completing and reviewing the collection of information. Send comments regarding this burden estimate or any other aspect of this collection of information. including suggestions for reducing this burden. to Washington Headquarters Services. Directorate for information Operations and Reports. 12 is Jefferson Davis Highway, Suite 1204. Arlington, VA 22202-4302, and to the Office of Management and Budget. Paperwork Reduction Project (0704-0 188), Washington, DC 20503.

\begin{tabular}{|l|c|c|c|}
\hline 1. AGENCY USE ONLY (Leave blank) & $\begin{array}{c}\text { 2. REPORT DATE } \\
\text { April 1995 }\end{array}$ & $\begin{array}{c}\text { 3. REPORT TYPE AND DATES COVERED } \\
\text { Technical Memorandum }\end{array}$ \\
\hline
\end{tabular}

4. TITLE AND SUBTITLE

5. FUNDING NUMBERS

Separation of Crack Extension Modes in Orthotropic Delamination Models

WU 505-63-50-04

\section{AUTHOR(S)}

Jack L. Beuth

7. PERFORMING ORGANIZATION NAME(S) AND ADDRESS(ES)

NASA Langley Research Center

Hampton, VA 23681-0001

8. PERFORMING ORGANIZATION REPORT NUMBER

9. SPONSORING/MONITORING AGENCY NAME(S) AND ADDRESS(ES)

National Aeronautics and Space Administration

Washington, DC 20546-0001
10. SPONSORING / MONITORING AGENCY REPORT NUMBER

NASA TM-109180

11. SUPPLEMENTARY NOTES

Work performed while author was at NASA Langley under the NASA/American Society for Education (ASEE)

Summer Faculty Fellowship Program (1994).

Beuth: Carnegie Mellon University, Pittsburgh, PA.

12a. DISTRIBUTION/AVAILABILITY STATEMENT 12b. DISTRIBUTION CODE

Unclassified - Unlimited

Subject Category 24

\section{ABSTRACT (Maximum 200 words)}

In the analysis of an interface crack between dissimilar elastic materials, the mode of crack extension is typically not unique, due to oscillatory behavior of near-tip stresses and displacements. This behavior currently limits the applicability of interfacial fracture mechanics as a means to predict composite delamination. The Virtual Crack Closure Technique (VCCT) is a method used to extract mode I and mode II energy release rates from numerical fracture solutions. The mode of crack extension extracted from an oscillatory solution using the VCCT is not unique due to the dependence of mode on the virtual crack extension length, $\Delta$.

In this work, a method is presented for using the VCCT to extract $\Delta$-independent crack extension modes for the case of an interface crack between two in-plane orthotropic materials. The method does not involve altering the analysis to eliminate its oscillatory behavior. Instead, it is argued that physically reasonable, $\Delta$-independent modes of crack extension can be extracted form oscillatory solutions. Knowledge of near-tip fields is used to determine the explicit $\Delta$ dependence of energy release rate parameters. Energy release rates are then defined that are separated from the oscillatory dependence on $\Delta$. A modified VCCT using these energy release rate definitions is applied to results from finite element analyses, showing that $\Delta$-independent modes of crack extension result. The modified technique has potential as a consistent method for extracting crack extension modes from numerical solutions. The $\Delta$-independent modes extracted using this technique can also serve as guides for testing the convergence of finite element models. Direct applications of this work include the analysis of planar composite delamination problems, where plies or debonded laminates are modeled as in-plane orthotropic materials.

\section{SUBJECT TERMS}

Delamination; Orthotropic; Interfacial fracture mechanics; Mode of crack tension; Virtual crack closure technique; Energy release rate 15. NUMBER OF PAGES

25 16. PRICE CODE

$\mathrm{AO} 3$

\begin{tabular}{l|c|}
\hline 17. SECURITY CLASSIFICATION & 18. $\begin{array}{c}\text { SECURITY CLASSIFICATION } \\
\text { OF REPORT } \\
\text { Unclassified }\end{array}$ \\
Unclassified
\end{tabular}

19. SECURITY CLASSIFICATION OF ABSTRACT
20. LIMITATION OF ABSTRACT 

